\title{
La participación ciudadana en el futuro de Europa: instrumentos y oportunidades para promover un espacio público europeo
}

\section{Citizen Participation in the Future of Europe: Instruments and Opportunities to Promote a European Public Sphere}

\author{
Beatriz Pérez de las Heras ${ }^{1}$ \\ Universidad de Deusto (España)
}

Recibido: 03-09-20

Aceptado: 06-10-20

\section{Resumen}

Como organización internacional de integración y proyecto de unión política, la Unión Europea (UE) ha introducido en la última década diversos mecanismos de participación ciudadana con el fin de reforzar su legitimidad democrática y apoyo popular. A pesar de su relevancia, estos instrumentos no han tenido un efecto transformador visible en las políticas y normativas europeas. La Conferencia sobre el Futuro de Europa, como nuevo foro participativo, aspira a dar ese salto cualitativo en el modelo de gobernanza, canalizando las propuestas colectivas resultantes de este proceso a través del ciclo institucional normativo. Esta nueva dinámica de innovación política requerirá cambios sistémicos en la representación y participación ciudadana. Sólo así podrá configurarse un espacio público genuinamente europeo, en el que los ciudadanos se identifiquen como demos de una comunidad política supranacional.

\footnotetext{
${ }^{1}$ (beatriz.perez@deusto.es) Doctora en Derecho (Universidad de Deusto). DESS y DEA en Droit Communautaire (Centre Européen Universitaire de Nancy, France). Catedrática, Profesora de Derecho de la Unión Europea y Titular de la Cátedra Jean Monnet en Integración Europea. Algunas publicaciones recientes: "The European Union in International Investment Governance: a Hybrid Approach to Dispute Settlement", Romanian Journal of European Affairs, vol. 18, n², 2018, pp.77-93;“La Agenda de Legislar Mejor como Eje de Gobernanza Democrática en la Unión Europea: impacto y potencialidades para las entidades sub-estatales", Revista General de Derecho Administrativo, $\mathrm{n}^{\circ}$ 50, 2019, pp. 1-39; "Climate security in the European Union's foreign policy: addressing the responsibility to prepare for conflict prevention", Journal of Contemporary European Studies, 2020, vol. 28, no 3, pp. 335-347.

ORCID: https://orcid.org/0000-0003-4402-2925.
} 
Palabras-clave: espacio público europeo, participación ciudadana, democracia deliberativa, Conferencia sobre el Futuro de Europa

\begin{abstract}
As an international organization of integration and project of political union, the European Union (EU) has introduced various mechanisms of citizen participation in the last decade with the aim of reinforcing its democratic legitimacy and popular support. Despite their relevance, these instruments have not had a visible transformative effect on European policies and laws. The Conference on the Future of Europe, as a new participatory forum, aspires to make that qualitative leap in the governance model, by channeling the collective proposals resulting from this process through the regulatory institutional cycle. This new dynamic of political innovation will require systemic changes in citizen representation and participation. It is in this way that a genuinely European public sphere can be shaped so that citizens may identify themselves as the demos of a supranational political community.
\end{abstract}

Key-words: European public sphere, citizen participation, deliberative democracy, Conference on the Future of Europe.

\title{
1. Introducción
}

A lo largo de la última década, tanto la Unión Europea (UE), como sus Estados miembros han sido testigos de movimientos de retroceso democrático, que se han evidenciado principalmente en la fuerte irrupción de fuerzas populistas y dinámicas gubernamentales antiliberales. La crisis de la eurozona, las medidas de austeridad presupuestaria y el incremento de las desigualdades sociales entre los países miembros explican en gran parte este proceso de erosión democrática, que hace temer la sostenibilidad del proyecto político europeo como ideal de paz y de prosperidad en Europa (Wilkison 2015; Pérez de las Heras 2015).

Por otra parte, el proceso de integración europea se ha ido construyendo tradicionalmente de arriba abajo. Su desarrollo a lo largo del tiempo ha dado por supuesta una ínsita legitimidad democrática para adoptar decisiones. Esta dinámica de evolución ha acabado por deslegitimar la idea misma de unidad política europea, al tiempo que ha alimentado la narrativa populista sobre la incapacidad de las instituciones europeas para garantizar bienes públicos esenciales, como el empleo, la vivienda o la seguridad (Muller 2012: 44). Si la UE desea sobrevivir como un proyecto común atractivo y creíble, es necesaria 
una vuelta de tuerca completa con respecto a la legitimidad subyacente en el modelo de gobernanza europea. Principalmente, resulta ineludible revisar los mecanismos de participación ciudadana para generar un entorno democrático transnacional, que facilite la configuración de un espacio público europeo donde los ciudadanos de distinta procedencia cultural, social, histórica y política puedan identificarse como miembros de una comunidad supranacional democrática (Peters 2013; Beetz and Rossi 2017; Walter 2017: 27-49).

La idea de un espacio público europeo ha sido frecuentemente unida a la de una identidad europea, entendida ésta como un sentimiento de pertenencia a una comunidad política supranacional (Eriksen 2005). En las últimas dos décadas, la cuestión del espacio público europeo ha sido también vinculada al debate sobre el déficit democrático de la UE. Así, por ejemplo, la Comisión Europea ha intentado abordar esta carencia y construir un espacio público europeo con iniciativas como la del Plan D para la Democracia, el Diálogo y el Debate (Plan D), o la declaración de 2013 como el Año Europeo de los Ciudadanos ${ }^{2}$. En los documentos de ambas propuestas, el ejecutivo europeo incide en la necesidad de desarrollar un espacio público europeo como un entorno donde "los ciudadanos dispongan de información y de instrumentos para participar activamente en el proceso de adopción de decisiones, sintiéndose partícipes del proyecto europeo"3. Ambas iniciativas abogaban, por tanto, por una democracia deliberativa, esto es, por la implicación activa de los ciudadanos en los procesos decisorios de la UE. Sin embargo, ninguno de estos impulsos tuvo el resultado esperado, ya que desembocaron en más debate ciudadano sobre temas europeos, pero no en deliberación (Van de Putte 2014).

Estas experiencias demuestran que debate y diálogo no siempre conducen a deliberación, que es un concepto más amplio. En términos generales, puede decirse que la deliberación es la capacidad de influir y de transformar las opciones y preferencias previamente expresadas por un actor o una institución. La democracia deliberativa parte de la idea de que lo es común tiene que decidirse en público y de modo participativo, y no a priori (Silva 2017).

La democracia deliberativa sigue siendo todavía un reto pendiente a nivel de la UE. Para abordarlo, nuevos mecanismos de participación ciudadana directa se han habilitado en los últimos años. Entre los más destacados, la iniciativa ciudadana europea y las consultas públicas sobre las políticas y propuestas de actos jurídicos europeos. Sin embargo, a pesar de los avances observados en términos de implicación ciudadana, puede afirmarse que la UE sigue careciendo por el momento de una infraestructura deliberativa sólida. El último impulso

\footnotetext{
${ }^{2}$ Respectivamente, Comisión Europea, "Contribución de la Comisión al periodo de reflexión y más allá: Plan D de democracia, diálogo y debate”, COM (2005) 494 final de 13 de octubre de 2005; "La Comisión Europea pone en marcha el año europeo de los ciudadanos", comunicado de prensa IP/13/2, 1 de enero de 2013.

${ }^{3}$ Comisión Europea, 2. Objetivos del Plan D, op.cit., p.3.
} 
para responder a esta carencia democrática es la Conferencia sobre el Futuro de Europa, un proceso de dos años propuesto por la Comisión de Ursula von der Leyen, en el que los ciudadanos podrán debatir y decidir sobre los grandes retos y preocupaciones que afronta la UE (von der Leyen 2019: 21). En términos de concepción, la Conferencia sobre el Futuro de Europa se presenta ante la opinión pública como un nuevo modelo de reformar Europa y un intento de revitalizarla democráticamente. Independientemente de las reformas de fondo que puedan resultar, la UE se juega su credibilidad en este proceso. En efecto, si después de las expectativas que ha generado sobre el liderazgo ciudadano con la convocatoria de este foro, la UE no llegara a cumplir su compromiso de incorporar y plasmar en resultados normativos las propuestas acordadas, este desajuste abocaría a una pérdida irremediable de confianza por parte de los ciudadanos en las reglas democráticas, pudiendo comprometer la continuidad misma del proyecto europeo.

Este artículo analiza el alcance de la participación de los ciudadanos en la construcción política de Europa. En particular, se adentra primeramente en los actuales mecanismos más relevantes de democracia participativa, valorando su impacto en los procesos legislativos y políticos de la UE. Partiendo de este análisis, el estudio se detiene en las perspectivas que ofrece la Conferencia sobre el Futuro de Europa, como nueva dinámica de gobernanza democrática y de reforma de los Tratados. Aún pendiente de desarrollarse este proceso, el estudio valora su potencialidad como foro estable de democracia deliberativa, que puede abrir el camino hacia un espacio público europeo.

\section{Mecanismos de democracia participativa en la UE: hacia fórmulas mejoradas y más inclusivas}

La iniciativa ciudadana europea y las consultas públicas sobre las propuestas normativas constituyen los mecanismos más relevantes de democracia directa introducidos por la UE en la última década. La utilización de ambos instrumentos se desarrolla a través de herramientas digitales de alcance trasnacional. A pesar de su potencialidad para reducir el déficit democrático de la UE, ambos mecanismos, por diferentes motivos, arrojan un bajo impacto en los procesos legislativos, lo que ha llevado recientemente a la introducción de ajustes para mejorarlos. Por otra parte, el proceso de digitalización creciente de la economía y sociedad europea ofrece también la posibilidad de introducir nuevas herramientas de comunicación e interacción ciudadana, que pueden contribuir a la creación de un espacio público europeo.

2.1. La iniciativa ciudadana europea y las consultas públicas: la democratización progresiva de los procesos normativos en la UE 
La iniciativa ciudadana europea representa el primer instrumento de democracia participativa trasnacional, sin precedente ni parangón a nivel mundial. Constituye un mecanismo único dentro también de la propia UE ya que, a diferencia de otros instrumentos participativos, como el derecho de petición al Parlamento Europeo, que sólo permite a los ciudadanos expresar sus preferencias en forma de solicitudes o quejas, o las mismas consultas públicas sobre propuestas previamente elaboradas por la Comisión Europea, la iniciativa europea faculta a los ciudadanos a dirigir sus propias propuestas legislativas directamente a la Comisión Europea.

Esta prerrogativa ciudadana se consagra en el párrafo 4 del artículo 11 del Tratado de la Unión Europea (TUE), introducido por el Tratado de Lisboa (2009). En concreto, esta disposición establece que:

\begin{abstract}
"Un grupo de al menos un millón de ciudadanos de la Unión, que sean nacionales de un número significativo de Estados miembros, podrá tomar la iniciativa de invitar a la Comisión Europea, en el marco de sus atribuciones, a que presente una propuesta adecuada sobre cuestiones que estos ciudadanos estimen que requieren un acto jurídico de la Unión para fines de la aplicación de los Tratados".
\end{abstract}

Poco después, el Reglamento UE 211/2011 de 16 de febrero de 2011 precisaba las condiciones y los aspectos operativos de su ejercicio ${ }^{4}$. Las primeras iniciativas se registraron en 2012. Desde entonces, el mecanismo ha sido objeto de continuas críticas, referidas tanto a los aspectos técnicos y burocráticos, como a los escasos resultados obtenidos. Ocho años después de su puesta en práctica, persisten todavía ciertas deficiencias en su procedimiento y función misma, a pesar incluso de haber sido revisado recientemente, por lo que su impacto como instrumento de democracia deliberativa continúa siendo prácticamente nulo (Alander y von Ondarza 2020: 284).

En efecto, sólo cinco de las 70 iniciativas que por el momento (septiembre 2020) han conseguido reunir el número de firmas necesarias han completado el procedimiento establecido 5 . De esas cinco iniciativas, sólo una, la registrada bajo el título Right 2 Water, tuvo éxito, en el sentido de que condujo a una propuesta de modificación de la Directiva 98/83/CE relativa a la calidad de las aguas destinadas al consumo humano, aunque la Comisión Europea tardó casi cinco años en tramitar la propuesta, que está todavía en curso $^{6}$. Con respecto a otras dos iniciativas, la Comisión también decidió introducir pequeños ajustes

\footnotetext{
${ }^{4}$ Reglamento UE 211/2011 de 16 de febrero de 2011 del Parlamento Europeo y del Consejo de 16 de febrero de 2011 sobre la iniciativa ciudadana, DOUE L 65/1 de 11 de marzo de 2011.

${ }^{5}$ Véase al respecto la información disponible en https://europa.eu/citizens-initiative/home es

${ }^{6}$ Comisión Europea, "Propuesta de Directiva del Parlamento Europeo y del Consejo relativa a la calidad de las aguas destinadas al consumo humano (texto refundido)", COM (2017) 753 final de 1 de febrero de 2018 .
} 
en la normativa existente, aunque sin incorporar todos los aspectos requeridos por los promotores, mientras que consideró que no era necesario introducir cambio o innovación jurídica alguna con relación a las otras dos iniciativas (Anglmayer 2015: 24-26).

El escaso impacto que han tenido las iniciativas que han conseguido reunir el número de apoyos necesarios demuestra que el procedimiento no ha respondido a las expectativas iniciales. En particular, el aspecto más criticado ha sido la facultad que tiene la Comisión de rechazar las iniciativas en base a los criterios de admisibilidad establecidos por el Reglamento UE 211/2011 (art. 4). Concretamente, casi todas las decisiones de rechazo de la Comisión se basan en el argumento de que la propuesta cae "manifiestamente fuera del ámbito de competencias de la Comisión para presentar una propuesta de un acto jurídico para los fines de aplicación de los Tratados"(art. 4.2b) (Russack 2018: 16-18). Este argumento reiterado ha generado una enorme frustración por parte de los promotores, al tiempo que ha suscitado dudas sobre la voluntad de la Comisión de tomarse en serio la iniciativa ciudadana (Organ 2014: 425).

En 2019 la UE acometió una revisión del mecanismo de iniciativa ciudadana, con el propósito de mejorar los aspectos funcionales del mismo. Como resultado, el nuevo Reglamento UE 2019/788 de 17 de abril de 2019 entró en vigor el 1 de enero de 2020, derogando el Reglamento UE 211/20117. La nueva disposición flexibiliza algunos de los problemas prácticos observados en el anterior procedimiento. Así, la fecha para el inicio de la recogida de firmas puede ahora ser fijada por los organizadores dentro de los 6 meses siguientes al registro de la iniciativa y todos los ciudadanos, independientemente de su lugar de residencia, pueden firmar la propuesta. Con respecto a los criterios de admisibilidad, recogidos en el artículo 6 del nuevo Reglamento, se contempla ahora la posibilidad de un registro parcial de la iniciativa, así como la revisión de la misma, en el caso de que no supere el test de admisibilidad (arts. 6.3c y 4). Finalmente, se prevé que los organizadores puedan exponer su iniciativa en el plazo de tres meses desde su presentación en audiencia pública ante el Parlamento Europeo (art. 14.2), que además asumirá el seguimiento de las iniciativas que hayan prosperado sobre la base de la comunicación que la propia Comisión Europea debe remitirle (arts. 15.2 y 16).

Es todavía pronto para valorar si la aplicación del nuevo Reglamento UE 2019/788 va a solventar los problemas técnicos y funcionales observados bajo la normativa anterior. No obstante, lo que está claro por el momento es que los ajustes introducidos no abordan la cuestión de la función y el propósito mismo de la iniciativa ciudadana. En efecto, la experiencia evidencia claramente una divergencia de percepciones entre los organizadores y la Comisión. Para los primeros, la iniciativa ciudadana es un instrumento para generar cambio político

\footnotetext{
${ }^{7}$ DOUE L130/55 de 17 de mayo de 2019.
} 
e innovación jurídica. Para la Comisión Europea, es un mero mecanismo que puede influir en su agenda normativa, pero cuyo propósito principal es llamar la atención sobre ciertas cuestiones (Russack 2018: 21). Por tanto, mientras la iniciativa ciudadana siga contemplándose como un instrumento que compite con el poder de iniciativa legislativa de la Comisión, el impulso ciudadano de proponer nueva legislación continuará considerándose como una mera forma de limitar las prerrogativas de la Comisión.

Además, a falta de una reforma formal de los Tratados, la Comisión continuará teniendo la última palabra sobre la elaboración o no de una propuesta legislativa en respuesta a una iniciativa ciudadana. Esta preponderancia institucional sólo podría compensarte con una mayor implicación del Parlamento Europeo en el seguimiento de las iniciativas ciudadanas que hayan reunido el número requerido de firmas. Como se ha analizado anteriormente, el nuevo Reglamento UE 2019/788 refuerza de algún modo ese seguimiento parlamentario y, además, el propio Parlamento se ha comprometido a debatir en sesión plenaria las iniciativas ciudadanas, conforme a una reciente modificación de sus reglas de procedimiento interno ${ }^{8}$. Sin embargo, para dar más efectividad a este avance, sería relevante que la institución parlamentaria acompañara el debate con una votación que, aunque no fuera vinculante, serviría para presionar a la Comisión a que responda en un sentido determinado. Esta mayor presencia del Parlamento Europeo en el procedimiento contribuiría, además, a entrelazar más estrechamente la democracia representativa y la participativa en la UE (Boucher et al.2019: 12).

Las consultas públicas en los procesos legislativos y de elaboración de políticas constituyen también otro instrumento valioso de democracia participativa en la UE. En los últimos cinco años, en particular, las consultas públicas se han transformado en un elemento esencial de una nueva cultura legislativa, más abierta y participativa, propiciada por la Agenda de Legislar Mejor ${ }^{9}$. Sin embargo, su relevancia supera el simple interés de mejorar la calidad del Derecho de la UE, ya que las consultas públicas abren los procesos normativos más allá del marco institucional, generando en los ciudadanos un sentimiento de coautoría legislativa, especialmente si sus opiniones y propuestas se ven reflejadas en el acto jurídico final. Por tanto, el mecanismo representa una valiosa inversión en democracia trasnacional, ya que refuerza la legitimidad democrática de la UE y contribuye a la aceptación popular de su normativa.

Al día de hoy, a diferencia de otros elementos que conforman la Agenda de Legislar Mejor como, por ejemplo, las evaluaciones de impacto, para las

\footnotetext{
${ }^{8}$ En concreto, los artículos 222 y 230 del Reglamento interno del Parlamento Europeo 2019-2024, disponible en https:/www.europarl.europa.eu/doceo/document/RULES-9-2019-07-02 ES.pdf

9 Comisión Europea, "Legislar mejor para obtener mejores resultados. Un programa de la UE", COM (2015) 215 final de 19 de mayo de 2015.
} 
que no hay base jurídica obligatoria, las consultas públicas no son una opción para la Comisión Europea, sino un mandato constitutivo, consagrado en los párrafos 2 y 3 del artículo 11 del TUE. En cumplimiento de este requisito legal, la Comisión Europea está obligada a abrir estas consultas públicas cuando prepara, en concreto, las propuestas de actos jurídicos, así como la revisión de la legislación existente, las comunicaciones de carácter consultivo y los Libros Verdes $^{10}$.

Actualmente, las consultas públicas y la posibilidad de aportar opiniones se canalizan a través de un único sitio web denominado "Contribuir al proceso legislativo" 11 . A través de esta canal y durante cuatro semanas, los interesados pueden ofrecer sus comentarios sobre las evaluaciones de impacto que acompañan a las nuevas iniciativas y las hojas de ruta, que incluyen evaluaciones y controles de adecuación de la normativa en vigor. A continuación, pueden participar también en las consultas públicas sobre las evaluaciones de impacto de las nuevas iniciativas legislativas ya formuladas, así como sobre las evaluaciones y controles de adecuación de la normativa existente, durante otro plazo de 12 semanas. En este último supuesto, los ciudadanos pueden transmitir sus sugerencias sobre cómo simplificar la legislación en vigor. Estas contribuciones de la opinión pública europea son tenidas en cuenta por un grupo de expertos, que conforman lo que se denomina actualmente la "Plataforma Preparados para el Futuro", encargada de ayudar a la Comisión a simplificar la legislación vigente ${ }^{12}$.

Las consultas públicas, abiertas a la participación de cualquier ciudadano, sin que sea necesariamente un experto en la materia, representan, sin duda, una dinámica valiosa de gobernanza multinivel, que mejora los procesos legislativos de la UE de abajo arriba, reforzando su legitimidad democrática como legislador supranacional. Sin embargo, la experiencia observada en los últimos cinco años revela, también en el caso de este mecanismo, algunas debilidades relativas, principalmente, a su accesibilidad y transparencia.

En efecto, una insuficiencia importante es la referida a los idiomas en los que se desarrollan estas consultas públicas. Normalmente, las propuestas y actos jurídicos objeto de consulta, así como los formularios de respuesta, se publican en inglés y, acaso también, en francés y alemán, utilizándose únicamente todas las lenguas oficiales de la UE cuando se trata de grandes iniciativas del programa anual de la Comisión Europea. En la práctica, no obstante, los interesados pueden enviar sus opiniones en su lengua materna,

\footnotetext{
${ }^{10}$ Comisión Europea, "Better regulations guidelines”, SWD (2017) 350 de 7 de julio de 2017, p. 71.

${ }^{11}$ https://ec.europa.eu/info/law/contribute-law-making_es

12 Desde 2020, esta plataforma reemplaza la Plataforma REFIT que, con el mismo propósito, trabajó de 2015 a 2019. Véase la información disponible en https://ec.europa.eu/info/law/law-makingprocess/evaluating-and-improving-existing-laws/refit-making-eu-law-simpler-and-less-costly/fitfuture-platform-f4f_en
}

Araucaria. Revista Iberoamericana de Filosofia, Politica, Humanidades y Relaciones Internacionales, año $22, \mathrm{n}^{\circ} 45$. Tercer cuatrimestre de 2020. Pp. 81-102. ISSN 1575-6823 e-ISSN 2340-2199 https://dx.doi.org/10.12795/araucaria.2020.i45.04 
pero esta selección lingüística constituye de entrada una dificultad adicional y un factor de exclusión que contraviene o, cuando menos, rebaja el principio de democracia participativa (Pérez de las Heras 2019: 12-13). Por tanto, con objeto de hacer más inclusivo el mecanismo, la Comisión debería garantizar que todos los cuestionarios y documentos de consulta estén accesibles en todas las lenguas oficiales de la UE, pudiendo acaso incluirse en los cuestionarios generales un apartado más específico para expertos y especialistas ${ }^{13}$.

Otro aspecto a mejorar es la calidad de las respuestas proporcionadas por la Comisión Europea. Conforme a las orientaciones generales que implementan la Agenda de Legislar Mejor, la Comisión tiene que publicar dos tipos de informes, tras el cierre del proceso de consultas. El primero es un informe donde se recoge un resumen fáctico del proceso (perfil de los interesados, número de participantes y su distribución geográfica, entre otros aspectos). El segundo es un informe sinóptico, en el que la Comisión debe proporcionar un análisis detallado de las respuestas y contribuciones recibidas, informando a los ciudadanos sobre cómo pretende incorporar sus aportaciones y explicando las razones por las cuales no pueden ser tenidas en cuenta ${ }^{14}$. En la práctica, la Comisión se demora habitualmente entre 6 y 9 meses en la elaboración de estos informes, la mayoría de los cuales sólo está disponible en inglés. Estas deficiencias han suscitado numerosas críticas por parte de los participantes en estos procesos, quienes con frecuencia declaran que no han recibido respuesta alguna de la Comisión, o que no saben cómo buscar los resultados de sus encuestas, ya que la Comisión ni siquiera les envía un email con el enlace a esta información, o que, simplemente, no pueden leer los informes porque están publicados en un solo idioma ${ }^{15}$.

Por tanto, las consultas públicas en los procesos legislativos y de elaboración de políticas representa un valioso instrumento constructivo de democracia deliberativa, pero necesita mejorarse para ser más inclusivo y transparente y permitir así un diálogo real y creíble entre los ciudadanos y las instituciones UE.

Las nuevas tecnologías pueden contribuir también a promover una participación ciudadana más amplia y activa, generar nuevas ideas y reforzar la legitimidad y confianza política.

13 European Court of Auditors, "Have your say: Commission's public consultations engage citizens, but fall short of outreach activities", Special Report 14 (2019), pp. 49-50.

${ }^{14}$ Comisión Europea, op cit, pp. 84 y 87.

15 European Court of Auditors, op. cit., pp. 41-45. 


\subsection{La democracia electrónica: posibilidades para la interacción} ciudadana en todo el proceso político europeo

El término de democracia electrónica se refiere al uso de las tecnologías de información y comunicación en la participación en los debates políticos y procesos de toma de decisiones (Aichholzer et al. 2018). El desarrollo de estas herramientas tecnológicas ha sido modesto por el momento en la UE, por lo que hay margen para su desarrollo y mejora. Como hemos analizado, mecanismos de participación electrónica como la iniciativa ciudadana o las consultas públicas presentan importantes debilidades que han limitado la capacidad de influencia de los ciudadanos en los procesos de elaboración de políticas y normativa europea.

En los últimos cinco años, la Comisión Europea ha lanzado varias iniciativas para promover la participación y el compromiso ciudadano en la vida pública europea a través de plataformas electrónicas. En este contexto, puede destacarse, por ejemplo, el Plan de Acción de la Administración Electrónica de la UE 2016-2020, cuyo objetivo prioritario es la transformación digital de las administraciones públicas como medio para empoderar a los ciudadanos ${ }^{16}$. Este Plan de Acción fue completado por la Declaración de Tallín, en la que los Estados miembros de la UE y los cuatro países de la Asociación Europea de Libre Comercio se comprometieron a mejorar sus administraciones públicas, proporcionar servicios transfronterizos, licitaciones electrónicas y una identificación electrónica ${ }^{17}$. En la misma línea, los servicios del Parlamento Europeo elaboraron en 2018 un informe sobre las perspectivas y potencialidades de la democracia electrónica en la UE (Korthangen et al. 2018).

Sin embargo, estas iniciativas han sido escasas y, en ningún caso, responden a una estrategia integral de democracia electrónica. Entre las insuficiencias observadas, los actuales instrumentos no contemplan canales de participación ciudadana en los procesos institucionales de adopción de decisiones, ni en la concepción de las políticas y medidas a adoptar. La Comisión y el Parlamento Europeo, instituciones implicadas en estos procesos y cercanas a los ciudadanos, podrían explorar esas formas de participación más amplia, tomando como inspiración algunas de las numerosas experiencias que se están desarrollando a nivel local en los Estados miembros. Así, plataformas digitales como la griega SynAthina para iniciativas ciudadanas, la finlandesa Kokeilum Paikka, que financia la generación de ideas para resolver problemas o la francesa Territoires Zéro Chòmeurs de Longue Durée, que capta fondos públicos para financiar

\footnotetext{
${ }^{16}$ Comisión Europea, "Plan de Acción de la Administración electrónica de la UE 2016-2020. Acelerar la transformación digital de la administración”, COM (2016) 179 final de 19 de abril de 2016.

17 "Tallin Declaration on eGovernment at the ministerial meeting during Estonian Presidency of the Council of the EU on 6 October 2017", disponible en https://ec.europa.eu/digital-single-market/ en/news/ministerial-declaration-egovernment-tallinn-declaration
}

Araucaria. Revista Iberoamericana de Filosofia, Politica, Humanidades y Relaciones Internacionales, año $22, \mathrm{n}^{\circ} 45$. Tercer cuatrimestre de 2020. Pp. 81-102. ISSN 1575-6823 e-ISSN 2340-2199 https://dx.doi.org/10.12795/araucaria.2020.i45.04 
nuevos empleos, son algunos ejemplos de inteligencia colectiva que propician la innovación política (Boucher 2020). Adaptadas al particular sistema de gobernanza europeo, este tipo de experiencias podría servir de referencia a la Comisión Europea para recabar ideas de los ciudadanos, antes de abrir el proceso de consultas públicas, lo que permitiría formular las distintas políticas y medidas en colaboración abierta con los ciudadanos desde el principio del proceso político. Esta mayor interacción no sólo estimularía la inteligencia colectiva popular, sino que la incorporación de las ideas resultantes al ciclo político y normativo europeo contribuiría a alinear mejor las instituciones supranacionales con las prioridades de los ciudadanos (Boucher et al. 2019:11).

Como herramienta tecnológica, la denominada "inteligencia artificial" podría servir para mejorar el carácter representativo de los procesos europeos de decisión ${ }^{18}$. Esta técnica, en concreto, es capaz de clasificar y analizar automáticamente miles de contribuciones recogidas en las plataformas de participación ciudadana, independientemente, además, de los idiomas de trabajo de dichas plataformas (Sebe, Muresan y Vas 2020: 337). Por el momento, la UE está en un proceso incipiente de promover el uso de la inteligencia artificial mediante la regulación del sector, lo que incluye la aplicación de principios éticos para garantizar la protección y el buen uso de los datos de los ciudadanos ${ }^{19}$.

Además de la Comisión Europea, el Parlamento Europeo también puede reforzar su carácter representativo aprovechando el potencial tecnológico. De hecho, en las últimas elecciones europeas de mayo de 2019, el Parlamento Europeo, junto a otras instituciones de la UE, lanzaron varias iniciativas mediante el uso de instrumentos digitales con el fin de atraer e implicar la sociedad civil en el debate electoral. Un ejemplo fue la campaña "Esta vez voto", desarrollada a través de una plataforma digital de gran alcance. Es difícil precisar hasta qué punto este despliegue tecnológico influyó en el comportamiento de voto de los ciudadanos, pero lo cierto es que las elecciones de 2019 fueron las que registraron el mayor índice de votantes de los últimos 20 años, lo que se debió principalmente a un incremento de participación de los jóvenes europeos ${ }^{20}$. No obstante, existen otros medios digitales por los que la institución parlamentaria puede facilitar también la participación ciudadana, esta vez, en los procesos políticos europeos. En este sentido, al igual que la Comisión, el Parlamento Europeo también podría recabar ideas de los ciudadanos, que a continuación podría canalizar a través de solicitudes de propuestas normativas a la Comisión,

18 No hay un concepto consensuado sobre la inteligencia artificial, pero comúnmente se entiende como aquella rama de la ciencia, ligada a la computación que, procesando los datos disponibles, identifica la decisión más idónea para alcanzar un fin concreto, emulando al ser humano en sus pensamientos y razonamientos (Canals Ametller 2019:2).

${ }^{19}$ Comisión Europea, "Libro Blanco sobre la inteligencia artificial. Un enfoque europeo orientado a la excelencia y la confianza", COM (2020) 65 final de 19 de febrero de 2020.

20 Parlamento Europeo, "Elecciones europeas 2019: participación récord impulsada por los jóvenes", nota de prensa, 24 de septiembre de 2019.

Araucaria. Revista Iberoamericana de Filosofia, Politica, Humanidades y Relaciones Internacionales, año $22, \mathrm{n}^{\circ} 45$. Tercer cuatrimestre de 2020. Pp. 81-102. ISSN 1575-6823 e-ISSN 2340-2199 https://dx.doi.org/10.12795/araucaria.2020.i45.04 
en base a la facultad que tiene atribuida a tal fin por el art. 225 del TFUE. Así mismo, interactuando online, el Parlamento podría apoyar la participación de los ciudadanos en las consultas públicas sobre las propuestas de la Comisión, ofreciendo explicaciones sobre los aspectos más técnicos y especializados. Igualmente, a través de su propia web, la institución parlamentaria podría publicar una breve ficha informática por cada acto jurídico adoptado, indicando el título del mismo, cómo se ha transpuesto al Derecho nacional (en el caso de Directivas) y el impacto esperado sobre la ciudadanía, todo ello en un lenguaje sencillo y accesible en todas las lenguas oficiales de la UE.

Otra modalidad de democracia digital consistiría en la introducción del voto electrónico en las elecciones europeas. A tal fin, las tecnologías denominadas "de cadena de bloques" (también conocidas como blockchain) podrían proporcionar la seguridad necesaria a los procesos electorales, permitiendo la emisión de los votos desde cualquier parte del territorio de la UE y garantizando que los votos sean efectivamente registrados, sin posibilidad de ser manipulados (Cordero Valdavida 2019) ${ }^{21}$. Bien utilizadas, este tipo de tecnologías podría aplicarse en realidad, no sólo a los procesos electorales, sino también a otras funciones públicas para garantizar una interacción más estrecha entre los ciudadanos y las instituciones a nivel europeo y nacional.

Los instrumentos de participación electrónica ofrecen, por tanto, una oportunidad para promover un espacio público europeo, al interconectar a los ciudadanos en torno a asuntos de interés común y contribuir a proporcionar un apoyo de base a las políticas de la UE. Por el momento, como hemos destacado, el componente digital de la agenda prodemocrática de la UE está escasamente desarrollado. Entre las orientaciones políticas de la actual Comisión Europea aparece "Una Europa adaptada a la era digital", pero el progreso hacia su realización no contempla la democracia digital, ni la potenciación de los instrumentos electrónicos de participación (von der Leyen: 14-15). Sin embargo, cara al proceso abierto sobre su futuro, es indudable que la UE necesita fomentar la experimentación con las técnicas digitales, ya que indudablemente constituirán una pieza instrumental clave de las dinámicas de participación ciudadana.

\footnotetext{
${ }^{21}$ La cadena de bloques es una estructura informatizada de datos en la que la información se agrupa en bloques a los que se añaden informaciones de otros bloques de la cadena anterior en una línea temporal, de modo que gracias a técnicas criptográficas los datos contenidos en un bloque sólo pueden ser rechazados o editados modificando todos los bloques posteriores. Se considera, por ello, una tecnología que genera confianza en la información y que contribuye a la transparencia (Cordero Valdavida 2019).
} 


\section{La Conferencia sobre el Futuro de Europa: ¿una oportunidad para reinventar la gobernanza democrática?}

En enero de 2020, la Comisión Europea presentó las grandes líneas de la Conferencia sobre el Futuro de Europa, como "un nuevo foro público para un debate abierto, inclusivo, transparente y estructurado con los ciudadanos sobre una serie de prioridades y desafíos fundamentales" ${ }^{22}$. En el punto de partida, este foro, compartido y participativo con la sociedad civil, se perfila como una nueva dinámica de gobernanza, de abajo arriba, en la construcción política de Europa. Uno de los objetivos de la Conferencia es, precisamente, mejorar la vida democrática de la UE y, en particular, la participación de los ciudadanos más allá del diálogo político y las elecciones europeas. Además, el momento actual de reflexión ofrece una ocasión propicia para revisar también otros aspectos de democracia representativa, que contribuirían a reforzar la legitimidad de la gobernanza europea, acortando la distancia entre los ciudadanos y las instituciones de la UE.

3.1. Más allá del debate político: hacia una estructura permanente de democracia deliberativa

El debate sobre el futuro de Europa (entendiendo este término como referido a la UE) no es nuevo. En concreto, desde marzo de 2017, con ocasión del $60^{\circ}$ aniversario de los Tratados de Roma y la solicitud oficial de retirada presentada por el Reino Unido, se ha asistido a un intenso debate sobre el futuro de la UE en múltiples foros e instancias europeas. El punto de partida de este proceso de reflexión fue el Libro Blanco sobre el Futuro de Europa, presentado por la Comisión Europea el 1 de marzo de $2017^{23}$. Entre 2017 y 2019, más de 1600 consultas y diálogos ciudadanos fueron organizados por la propia Comisión para debatir sobre los escenarios propuestos por este documento. En la primavera de 2019, antes de las elecciones europeas de mayo de 2019, fue el Presidente francés, Emmanuel Macron, quien lanzó la idea de una Conferencia sobre el Futuro de Europa, un nuevo foro que incluiría a los ciudadanos, así como a representantes de las instituciones europeas y de los Estados miembros, y que se encargaría de debatir y proponer todos los cambios que requiere el proyecto político europeo, incluida la reforma de los Tratados (Macron 2019). Finalmente, tras las elecciones europeas, a la luz del mayor índice de participación y de los resultados positivos obtenidos por las fuerzas políticas pro-europeas, la nueva Presidenta de la Comisión Europea acogió e

\footnotetext{
${ }^{22}$ Comisión Europea, "Perfilando la Conferencia sobre el Futuro de Europa", COM (2020) 27 final de 22 de enero de 2020, p.1.

${ }^{23}$ Comisión Europea, "Libro Blanco sobre el Futuro de Europa. Reflexiones y escenarios para la Europa de los veintisiete en 2025”, COM (2017) 2025 final de 1 de marzo de 2017.
} 
hizo suya la iniciativa francesa, incorporándola como una de sus orientaciones políticas y designando como responsable de este proceso a Dubravka Suica, Vice-Presidenta de la Comisión Europea sobre Democracia y Demografía.

La organización de un foro participativo de este calado, con ciudadanos de 27 Estados miembros, trabajando en 24 lenguas oficiales, es una experiencia inédita, no sólo en la UE, sino en el espectro democrático comparado. Nunca se ha hecho y todavía se están discutiendo las condiciones para llevarlo a cabo. Sin embargo, el impulso constituye indudablemente una oportunidad para implicar a los ciudadanos en la construcción de Europa y, por ende, para promover una UE más democrática.

Al mismo tiempo, la propuesta de von der Leyen se sitúa en la línea de una tendencia actual, que constituye al mismo tiempo un reto en términos de pulso democrático, como es la proliferación de múltiples y diversos procesos de participación ciudadana e iniciativas deliberativas que se observan en numerosos Estados europeos y países del mundo occidental24. Así, por ejemplo, el Consejo de los Ciudadanos, creado en febrero de 2019, en la comunidad germano parlante de Ostbelgien, en Bélgica, constituye el primero y más avanzado modelo de estructura permanente de democracia deliberativa en el contexto de la UE. Creado por una ley del Parlamento de esta comunidad, el Consejo de los Ciudadanos se ha transformado en una nueva institución democrática en la gobernanza regional, que complementa la cámara parlamentaria directamente elegida. Otros ejemplos de esta nueva ola de democracia deliberativa, que pugna por participar e influir en las decisiones políticas, son las Asambleas Ciudadanas en Polonia, el Consejo Social, Económico y Medioambiental en Francia o el Consejo de los Ciudadanos en Austria (Chwalisz 2019) ${ }^{25}$. A nivel de la UE, sin embargo, como hemos destacado, los diversos instrumentos participativos y foros deliberativos que se han puesto en marcha en los últimos años no han tenido hasta ahora una mayor influencia de los ciudadanos en la agenda política y legislativa de la UE. La Conferencia sobre el Futuro de Europa pretende, precisamente, aportar ese valor democrático añadido, incorporando las ideas y propuestas de la sociedad civil a las políticas y normativas europeas.

La estructura y el mandato de la Conferencia están todavía en fase de definición. En el contexto institucional de la UE, el Parlamento Europeo fue el primero en presentar su contribución oficial al debate sobre este proceso. En concreto, en su Resolución de 15 de enero de 2020 sobre la Conferencia sobre el Futuro de Europa ${ }^{26}$, la institución parlamentaria desgrana sus propuestas sobre

${ }^{24}$ Esta nueva práctica democrática se recoge en las recomendaciones de la Organización para la Cooperación y el Desarrollo Económico (OCDE), formuladas en el documento "Recommendation of the Council on Open Government", 14 de noviembre de 2017, C (2017) 140 y C7M (2017) 22.

25 Sobre estas experiencias, véase Claudia Chwalisz, "Reshaping European democracy. A new wave of deliberative democracy", Carnegie Europe Paper, noviembre 2019.

${ }^{26}$ Parlamento Europeo, "Resolución del Parlamento Europeo, de 15 de enero de 2020, sobre la proposición del Parlamento Europeo sobre la Conferencia sobre el Futuro de Europa" 2019/2990

Araucaria. Revista Iberoamericana de Filosofia, Politica, Humanidades y Relaciones Internacionales, año $22, \mathrm{n}^{\circ} 45$. Tercer cuatrimestre de 2020. Pp. 81-102. ISSN 1575-6823 e-ISSN 2340-2199 https://dx.doi.org/10.12795/araucaria.2020.i45.04 
la estructura, función y resultados de la Conferencia, de forma más precisa que el documento de la Comisión publicado cinco días después. En este sentido, para el Parlamento Europeo, el eje central de este proceso de dos años serían las ágoras ciudadanas, integradas por entre 200-300 ciudadanos elegidos al azar, y el Plenario de la Conferencia, compuesto por unos 250-300 miembros, representantes de las instituciones de la UE y de los parlamentos nacionales. A ellos se añadiría una Ágora de Jóvenes, de entre 16 a 25 años. Todo el proceso estaría conducido por un Comité Director, integrado por representantes de las tres instituciones de la UE y que se encargaría, a su vez, de designar un Consejo Ejecutivo de Coordinación, todos ellos con el apoyo de una secretaría ${ }^{27}$.

Los foros ciudadanos mantendrían la misma composición a lo largo del proceso de la Conferencia para garantizar la coherencia y consistencia de los debates, y cada ágora se centraría en un tema específico. La propia Resolución del Parlamento propone, a título orientativo, una lista concreta de temas ${ }^{28}$ :

-Valores europeos, derechos y libertades fundamentales.

-Aspectos democráticos e institucionales de la UE.

-Desafíos ecológicos y crisis climática.

-Justicia social e igualdad.

-Empleo y asuntos económicos, incluida la fiscalidad.

-Cambio digital.

-Seguridad y papel de la UE en el mundo.

Leyendo la Resolución del Parlamento Europeo, se extrae la impresión de que el centro principal de las negociaciones será el Plenario de la Conferencia y que las propuestas de las ágoras ciudadanas serán meramente incorporadas de algún modo al debate plenario en curso. Por tanto, no está claro por el momento cómo serán tomadas en cuenta las contribuciones de los ciudadanos. Lo coherente con un proceso de participación deliberativa, como pretende ser la Conferencia, es que, una vez que las ágoras ciudadanas hayan transmitido sus propuestas al Plenario, éste estudie, motive y explique la viabilidad o no de transformarlas en políticas o acciones jurídicas concretas. De este modo, algunas de las propuestas que resulten acogidas favorablemente por el Plenario podrían canalizarse a través de actos jurídicos institucionales, Reglamentos y Directivas principalmente, según el ámbito de competencia de la UE. Otras propuestas, aquellas que requieran cambios en la estructura institucional o en la distribución de competencias entre la UE y los Estados miembros, serían incorporadas a través de la reforma de los Tratados (Padberg 2020: 5-6).

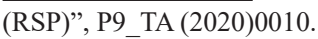

${ }^{27}$ Ibidem, pp.4-6.

${ }^{28}$ Ibidem, p. 3.

Araucaria. Revista Iberoamericana de Filosofia, Política, Humanidades y Relaciones Internacionales, año $22, \mathrm{n}^{\circ} 45$. Tercer cuatrimestre de 2020. Pp. 81-102. ISSN 1575-6823 e-ISSN 2340-2199 https://dx.doi.org/10.12795/araucaria.2020.i45.04 
Más allá del compromiso político asumido, la Comisión Europea, el Parlamento Europeo y el Consejo deberían concretar en un acuerdo interinstitucional cómo van a hacer operativos los resultados de la Conferencia sobre el Futuro de Europa, y evitar así que los nuevos esfuerzos de participación ciudadana fracasen como lo hicieron otros anteriores (Bialozyt y Le Quiniou 2020). Si la UE aspira verdaderamente a dar un salto cualitativo en su agenda prodemocrática, tendrá que incorporar y reflejar las contribuciones de este foro en sus procesos decisorios y normativos, no sólo en este momento concreto de reflexión, sino como un mecanismo permanente de democracia deliberativa.

\subsection{Una ocasión para innovar la agenda prodemocrática de la UE}

El proceso de debate abierto por la convocatoria de la Conferencia sobre el Futuro de Europa brinda a la UE un marco propicio para mejorar su narrativa prodemocrática y presentarse como un proyecto que incita a los ciudadanos a abordar conjuntamente los retos comunes, al tiempo que canaliza sus ideas y propuestas a través del ciclo institucional normativo. Un modelo de gobernanza democrática más fuerte estimula la inteligencia colectiva, generadora de nuevas ideas dirigidas a afrontar los grandes desafíos que afronta el proyecto político europeo.

No obstante, más allá de los procedimientos decisorios, una parte importante de esa nueva agenda prodemocrática debe incluir también cambios en el sistema de elección de los líderes y representantes políticos en la UE. Se incluyan o no estos cambios en el mandato de la Conferencia, la UE necesita, en particular, revisar el procedimiento de elección de la Presidencia de la Comisión Europea, de modo que evite la confusión ciudadana y la confrontación institucional que generó la designación de la actual Presidenta del ejecutivo europeo, tras las últimas elecciones europeas de 2019 (Boucher et al. 2019:5).

En efecto, a pesar de la euforia generada por la mayor participación y los resultados positivos de las fuerzas políticas pro europeas, no se siguió, a continuación, el procedimiento conocido como Spitzenkandidaten ${ }^{29}$, como se hizo por primera y única vez hasta ahora en las elecciones de 2014. Conforme a este sistema, los principales grupos políticos del Parlamento Europeo designan un candidato líder, que encabeza sus respectivas listas y que, de resultar la más votada a nivel de la UE, sería designado como Presidente de la Comisión Europea. Este procedimiento de cabezas de lista tiene como objetivo acercar el ejecutivo y el legislador europeo y, al mismo tiempo, las instituciones europeas y los ciudadanos, reforzando la legitimidad democrática del Presidente de

${ }^{29}$ Este procedimiento de cabezas de lista está tomado principalmente del modelo alemán, según el cual los ciudadanos no eligen directamente al jefe del Estado, sino a los miembros del Parlamento, quienes a su vez eligen al jefe del ejecutivo (Espaliú Berdud 2019: 174-179). 
la Comisión Europea. Sin embargo, tras las elecciones de mayo de 2019, el Consejo Europeo, de conformidad con el artículo 17.7 del TUE, designó a la alemana Ursula von der Leyen, que no era ninguno de los Spitzenkandidaten, ni había participado siquiera como elegible en los comicios europeos. El Consejo Europeo justificó la designación argumentando que ninguno de los candidatos a presidir la Comisión había obtenido una amplia mayoría. En esas circunstancias, optó por una candidata que reflejaba el equilibrio de intereses al que llegaron los distintos gobiernos de los Estados miembros. El Parlamento Europeo acabó aprobando el nombramiento, pero por un estrecho margen de votos, ya que fueron numerosos los miembros que expresaron su oposición, por considerar que el Consejo Europeo había ignorado un procedimiento destinado a reforzar la legitimidad y responsabilidad democrática de la Comisión Europea (Gros y Russack 2019).

Aprovechando el momento actual de debate y reforma, resulta oportuno establecer un procedimiento adecuado y jurídicamente vinculante para la designación de la Presidencia de la Comisión Europea, cara a las próximas elecciones europeas de 2024. En realidad, no se trataría de introducir un nuevo sistema de designación, sino de hacer que el proceso existente resulte más predecible y transparente. En este sentido, algunas propuestas apuestan por la implantación de dos fases en el procedimiento del Spitzenkandidaten. En una primera fase, los grupos políticos del Parlamento Europeo seguirían designando, en principio, su candidato a Presidente de la Comisión, como cabeza de lista. El candidato de la lista que obtuviera una amplia mayoría parlamentaria, aspecto éste último que debería estar establecido de antemano en términos de porcentaje, sería designado por el Consejo Europeo como nuevo Presidente del ejecutivo europeo. Por el contrario, si ningún candidato obtuviera una holgada mayoría, como ocurrió en las elecciones de 2019, el Consejo Europeo podría proponer su propio candidato, haya éste participado o no en el procedimiento del Spitzenkandidaten. Esta sería la segunda fase del sistema de designación (Russack 2020: 142-144).

Un acuerdo estableciendo estas nuevas normas en la implementación del procedimiento evitaría discusiones superfluas y garantizaría un equilibro de participación entre el Parlamento Europeo y el Consejo Europeo. Además, el nuevo sistema reflejaría la voluntad de los ciudadanos y de los gobiernos, como pilares fundamentales de la legitimidad de la UE.

Asociado a este procedimiento revisado de designación de la Presidencia de la Comisión, la introducción de una lista transnacional de candidatos al Parlamento Europeo constituiría, así mismo, un elemento nuevo de democracia representativa, que proporcionaría un nexo efectivo entre los ciudadanos y las instituciones de la UE. Dicha lista incluiría candidatos que se presentarían a nivel de toda la UE, pudiendo ser elegidos por los ciudadanos de cualquier 
Estado miembro. Este elemento nuevo supondría dotar a los ciudadanos de dos votos: uno, para los candidatos que se presenten a nivel nacional o regional, y el otro, para aquellos que lo hagan a nivel de la UE. La lista transnacional incluiría los candidatos a Presidente de la Comisión Europea de cada partido, de tal modo que los ciudadanos de todos los Estados miembros podrían votar por todos estos candidatos y no sólo por aquellos de su misma nacionalidad. Esta innovación electoral reforzaría la dimensión europea de los comicios al Parlamento Europeo, incitaría a los medios nacionales a dar más visibilidad a los grupos políticos europeos y a que los partidos nacionales pongan de relevancia sus afiliaciones transnacionales (Kovár, Sychra y Kratochvíl 2020).

El diseño institucional de una lista transnacional de candidatos europeos está pendiente de ser debatida y definida. La Comisión ha suscitado ya el debate en el contexto de la Conferencia sobre el Futuro de Europa. También el Parlamento, en su Resolución de 15 de enero, ha incluido los cabezas de lista y las listas trasnacionales como temas a considerar por la Conferencia ${ }^{30}$.

Así mismo, la introducción de un sistema electoral uniforme sigue siendo un tema pendiente. Esta carencia constituye uno de los principales obstáculos para el establecimiento de partidos políticos europeos a nivel de la UE. Por ahora, ninguna de las propuestas elaboradas en este sentido por el Parlamento Europeo han sido aprobadas. Un sistema electoral supranacional es un prerrequisito, no sólo para reforzar la representatividad y papel de los grupos políticos europeos, sino también para respetar el principio de igualdad de voto, actualmente transgredido por las regulaciones nacionales divergentes (Kovár, Sychra y Kratochvíl 2020: 121).

La revisión del procedimiento de designación del Presidente de la Comisión Europea y la introducción de una lista transnacional de candidatos, junto con un sistema electoral uniforme, son elementos que refuerzan la democracia representativa, acortan distancias entre el legislativo y ejecutivo europeo y fortalecen el vínculo entre los ciudadanos y el sistema político de la UE, contribuyendo a que estos últimos se identifiquen como miembros de un espacio público genuinamente europeo.

\section{Conclusión}

Una creciente demanda de participación pública recorre Europa y buena parte del mundo occidental. Nuevas fórmulas de participación civil están surgiendo y haciéndose evidentes en el contexto de los gobiernos locales y nacionales.

Como organización internacional de integración, la UE participa de esta tendencia de renovación democrática. En este sentido, hemos analizado como

\footnotetext{
${ }^{30}$ Parlamento Europeo, op. cit., apartado 8, p. 3.
} 
distintos mecanismos de participación ciudadana, de diferente alcance, han sido habilitados por la UE para promover la implicación de la sociedad civil en la construcción política europea. Sin embargo, el estudio ha revelado que, a pesar de la relevancia y la potencialidad de algunos de estos instrumentos, la experiencia de su utilización por los ciudadanos no ha tenido un impacto transformador relevante en las políticas y normativas europeas. Por otra parte, estos mecanismos de participación popular se han ido introduciendo de forma coyuntural y fragmentaria, sin responder a una agenda democrática integral y previsible.

La UE ha abierto en 2020 un nuevo proceso de reflexión sobre su futuro, en el que la reforma democrática seguirá siendo uno de los temas clave de debate. Pero, la renovación democrática en la UE necesitará algo más que meros retoques institucionales. A la luz del camino andado y de la experiencia observada, la UE necesita dotarse de un nuevo sistema de gobernanza en el que los ciudadanos puedan verdadera y activamente ser partícipes y en el que las ideas y propuestas colectivas puedan canalizarse a través del ciclo político institucional, reflejándose en último término en soluciones concretas a los problemas y preocupaciones sociales. Para alcanzar esta dinámica de innovación política, la UE necesitaría, entre otros aspectos, establecer un sistema permanente de participación ciudadana en la definición de políticas y adopción de legislación europea.

Sin prescindir de otras revisiones necesarias, la calidad del compromiso de participación colectiva es un componente esencial de la recuperación democrática en la UE. Por tanto, si la UE aspira a garantizar su sostenibilidad como proyecto de unión política, los líderes políticos y las instituciones europeas tienen que aceptar el inicio de una nueva etapa de democratización. La UE debe reinventar su modelo de gobernanza democrática con objeto de responder a los desafíos derivados de un complejo e inestable contexto mundial y europeo a través de la inteligencia colectiva y el apoyo popular. Es el reto que se traslada ahora a la Conferencia sobre el Futuro de Europa que, como nuevo foro constituyente, brinda la ocasión para acometer este salto sistémico.

Sin un espacio público europeo y unas nuevas bases constitutivas que reconcilien a los ciudadanos con el proyecto político europeo, la UE seguirá adoleciendo de una falta de legitimidad a los ojos de la gente. Por el contrario, es dentro de este espacio político y público común donde las fuerzas populistas y euroescépticas pueden ser superadas y realizarse la visión de Jean Monnet de crear un demos europeo que se identifique como miembro de una comunidad política europea. 


\section{Referencias bibliográficas:}

Aichholzer, Georg et al. 2018. "Prospects for e-democracy in Europe. Part I: Literature review", European Parliamentary Research Service, PE 603.213.

Alander, Nina y von Ondarza, Nicolai. 2020. "The European Citizens' initiative and its reform. Truly unique or the same old story?" [en Steffen Blockmans and Sophia Russack, eds.: Deliberative Democracy in the EU. Countering populism with participation and debate, Londres, Rowman \& Littlefield International], pp. 281-293.

Anglmayer, Irmgard. 2015. “The European Citizens' Initiative. The experience of the first three years. European implementation assessment", European Parliamentary Research Service, PE 536.343.

Beetz, Jan Pieter y Rossi, Enzo. 2017. “The EU's democratic deficit is a realistic key: multilateral governance, popular sovereignty and critical responsiveness" en Transnational Legal Theory, 1, pp. 22-41.

Bialozyt, Wojciech y Le Quiniou, Romain. 2020. "Europe's Deliberative Instruments: has the EU delivered?" [en Steffen Blockmans and Sophia Russack, eds.: Deliberative Democracy in the EU. Countering populism with participation and debate, Londres, Rowman \& Littlefield International], pp.313-329.

Boucher, Stephen et al. 2019. "Six ideas for rejuvenating European democracy", Carnegie Europe working paper, pp. 1-25.

Boucher, Stephen. 2020. "Can Europe be a catalyst for democratic innovation?", Carnegie Europe Commentary.

Canals Ametller, Dolors. 2019. "El proceso normativo ante el avance tecnológico y la transformación digital (inteligencia artificial, redes sociales y datos masivos" en Revista General de Derecho Administrativo, 50, pp. 1-26.

Cordero Valdavida, Magdalena. 2019. "Blockchain en el sector público, una perspectiva internacional" en Revista Vasca de Gestión de Personas y Organizaciones Públicas, 16, pp.16-34.

Chwalisz, Claudia. 2019. "Reshaping European democracy. A new wave of deliberative democracy", Carnegie Europe Paper.

Eriksen, Erik Oddvar. 2005. "An emerging European public sphere" en European Journal of Social Theory, 3, pp. 341-363.

Espaliú Berdud, Carlos. 2019. "Impulsar la participación de los ciudadanos como factor de legitimidad en el proyecto de la integración europea" en Cuadernos Europeos de Deusto, 60, pp. 157-186. 
Gros, Daniel y Russack, Sophia. 2019. "The nomination of von der Leyen: towards institutional balance in a reformed lead candidate process" en CEPS in brief, disponible en https:/www.ceps.eu/the-nomination-of-vonder-leyen/

Korthangen, Iris et al.. 2018. "Prospects for e-democracy in Europe. Part II: Case studies", European Parliamentary Research Service, PE 603.213.

Kovár, Jan, Sychra, Zdenek y Kratochvíl, Petr. 2020. “Alignment of national parties and European party federations" [en Steffen Blockmans and Sophia Russack, eds.: Deliberative Democracy in the EU. Countering populism with participation and debate, Londres, Rowman \& Littlefield International], pp.113-130.

Macron, Emmanuel. 2019. "Por un renacimiento europeo", disponible en https:// www.elysee.fr/emmanuel-macron/2019/03/04/por-un-renacimientoeuropeo.es

Muller, J-W. 2012. "Beyond militant democracy?” en New Left Review, 39, pp. $39-47$.

Organ, James. 2014. "Decommissioning direct democracy? A critical analysis of Commission decision making on the legal admissibility of European citizen initiative proposals" en European Constitutional Law Review, 3, 422-443.

Padberg, Stefan. 2020. "Towards the Conference on the Future of the EU", disponible en https:/www.transforming-europe.eu/fileadmin/user upload/pdf/Towards_The Conference_On_The_Future_V1.0.pdf

Pérez de las Heras, Beatriz. 2015. "Beyond the Markets: Citizen Participation and Social Progress for a more Popular and Inclusive Europe" [en Serge Champeau, Carlos Closa, Daniel Innenarity y Mario Poiares, eds.: The future of Europe. Democracy, Legitimacy and Justice after the Euro Crisis, Londres, Nueva York, Rowman \& Littlefield International], pp. 263-277.

Pérez de las Heras, Beatriz. 2019. "La Agenda de Legislar Mejor como eje de gobernanza democrática en la Unión Europea: impacto y potencialidades para las entidades subestatales" en Revista General de Derecho Administrativo, 50, pp.1-39.

Peters, Mayte. 2013. "The democratic function of the public sphere in Europe" en German Law Journal, 14, pp. 673-693.

Russack, Sophia. 2018. "Pathways for citizens to engage in EU policymaking" [en Steffen Blockmans and Sophia Russack, eds.: Direct Democracy in the EU. The myth of a citizens' Union, Londres, Rowman \& Littlefield International], pp. 9-41. 
Russack, Sophia. 2020. "How to appoint a Commission President" [en Steffen Blockmans and Sophia Russack, eds.: Deliberative Democracy in the EU. Countering populism with participation and debate, Londres, Rowman \& Littlefield International], pp.131-147.

Sebe, Mihai, Muresan, Bogdan y Vas, Eliza 2020. "How can technology facilitate citizen participation in the EU?" [en Steffen Blockmans and Sophia Russack, eds.: Deliberative Democracy in the EU. Countering populism with participation and debate, Londres, Rowman \& Littlefield International], pp. 333-346.

Silva, Sara. 2017. "La democracia deliberativa como herramienta de la sociedad civil” en Revista de Educación Social, 24, pp. 1206-1210.

Van de Putte, Thomas. 2014. "Discourses on democracy. The European year of citizens 2013 and the public sphere" en CEU eTD Collection, disponible en www.etd.ceu.hu/2014/putte thomas.pdf

Von der Leyen, Ursula. 2019. "Una Europa que se esfuerza por lograr más resultados. Mi agenda para Europa. Orientaciones políticas para la próxima Comisión Europea 2019-2024”, 2019.

Walter, Stefanie. 2017. EU citizens in the European Public Sphere. An analysis of EU news in 27 Member States, Springer VS.

Wilkison, Michael A. 2015."Authoritarian liberalism in the European constitutional imagination: second time as farce?" en European Law Journal, 3 (2015), pp. 313-339. 